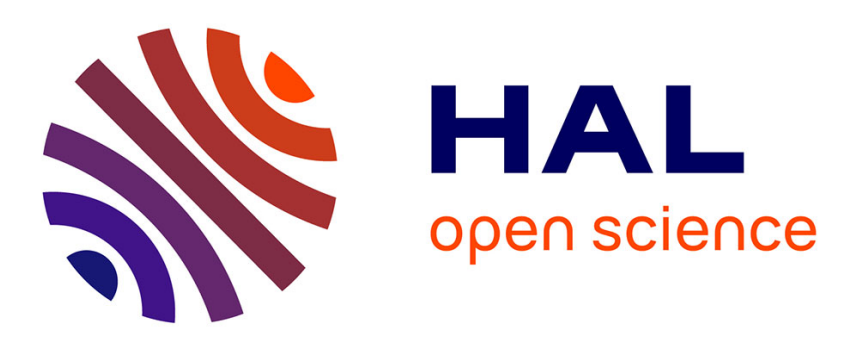

\title{
Electromagnetic Simulations of Solar Radio Emissions
}

\author{
Pierre Henri, A. Sgattoni, C. Briand, François Amiranoff, C. Riconda
}

\section{To cite this version:}

Pierre Henri, A. Sgattoni, C. Briand, François Amiranoff, C. Riconda. Electromagnetic Simulations of Solar Radio Emissions. Journal of Geophysical Research Space Physics, 2019, 124 (3), pp.1475-1490. 10.1029/2018JA025707 . insu-02057346

\section{HAL Id: insu-02057346 https://hal-insu.archives-ouvertes.fr/insu-02057346}

Submitted on 2 Oct 2019

HAL is a multi-disciplinary open access archive for the deposit and dissemination of scientific research documents, whether they are published or not. The documents may come from teaching and research institutions in France or abroad, or from public or private research centers.
L'archive ouverte pluridisciplinaire HAL, est destinée au dépôt et à la diffusion de documents scientifiques de niveau recherche, publiés ou non, émanant des établissements d'enseignement et de recherche français ou étrangers, des laboratoires publics ou privés. 


\section{JGR Space Physics}

\section{RESEARCH ARTICLE \\ 10.1029/2018JA025707 \\ Key Points: \\ - We single out the successive steps of the generation mechanisms of solar radio emissions \\ - We quantify the energy transferred from energetic particles to radio waves and show it is independent of energetic particles parameters \\ - We identify the directivity of the radio emissions and show its strong dependence on energetic particles parameters.}

Correspondence to:

P. Henri,

pierre.henri@cnrs-orleans.fr

Citation:

Henri, P., Sgattoni, A., Briand, C., Amiranoff, F., \& Riconda, C. (2019). Electromagnetic simulations of solar radio emissions. Journal of Geophysical Research: Space Physics, 124, 1475-1490. https://doi.org/10.1029/2018JA025707

Received 23 MAY 2018

Accepted 7 JAN 2019

Accepted article online 3 MAR 2019

Published online 15 MAR 2019

\section{Electromagnetic Simulations of Solar Radio Emissions}

\author{
P. Henri' ${ }^{1}$, A. Sgattoni ${ }^{2,3}$, C. Briand ${ }^{2}$, F. Amiranoff ${ }^{3}$, and C. Riconda ${ }^{3}$ \\ ${ }^{1}$ LPC2E, CNRS, Orléans, France, ${ }^{2}$ LESIA, Observatoire de Paris, PSL Research University, CNRS, Sorbonne Université, \\ UPMC Université Paris 06, Université Paris Diderot, Sorbonne Paris Cité, Meudon, France, ${ }^{3}$ LULI, Sorbonne Université, \\ UPMC Université Paris 06, École Polytechnique, CNRS, CEA, Paris, France
}

\begin{abstract}
Solar radio emissions are electromagnetic waves emitted in the solar wind as a consequence of electron beams accelerated during solar flares or interplanetary shocks such as interplanetary coronal mass ejections. Different physical mechanisms have been suggested to describe their origin. A good understanding of the emission process would enable to infer the kinetic energy transferred from accelerated electrons to radio waves. Even if the electrostatic case has been extensively studied, full electromagnetic simulations were attempted only recently. In this work, we report large-scale 2D3V electromagnetic particle-in-cell simulations that enable to identify the generation of both electrostatic and electromagnetic waves originated by a succession of plasma instabilities. They confirm that an efficient mechanism to generate solar radio emissions close to $T_{2 f}$, the harmonic of the plasma frequency, is a multistage model based on a succession of nonlinear three-wave interaction processes. Through a parametric study of the electron beam parameters, we show that (i) the global efficiency of the multistep conversion mechanism from the electron beam kinetic energy to the $T_{2 f}$ radio wave is independent of the beam parameters, approximately $10^{-5}$ in all tested configurations, while (ii) the directivity of the electromagnetic radio wave strongly depends on the origin electron beam. Those results represent a step forward toward the use of solar wind radio emissions, observed remotely, as a diagnostic for the properties of the electron beam located at the source of the radio emission, and therefore to eventually better characterize remotely electron acceleration mechanisms in space regions not directly accessible to in situ measurements.
\end{abstract}

\section{Introduction}

The interplanetary radio emissions in the range $10 \mathrm{kHz}$ to $100 \mathrm{MHz}$ are the signature of 10 - to $100-\mathrm{keV}$ electron beams propagating in the interplanetary medium after solar eruptions or ahead of interplanetary shocks. While the detection of electron beams requires that they cross the spacecraft, remote detection of widely spread radio waves is easy either by ground-based antennas or by spacecraft. The radio waves are thus very powerful to diagnose the electron beams (e.g., velocity and density) and interplanetary medium characteristics (e.g., density fluctuations).

A consensus exists on the fact that the electromagnetic (hereafter EM) radiation results from the conversion of electron plasma waves. The debate mostly arises when trying to identify the conversion mechanism(s) or to quantify the scattering of the EM radiation into the interplanetary medium (with the consequences on the determination of the characteristics of the radiation source). Weak turbulence conditions seem to prevail in the solar wind. This means that the electric energy in the Langmuir waves is much smaller than the electron thermal energy $\left(E_{L}^{2} / 4 \pi n_{0} T_{e} \ll 10^{-2}\right)$. In this weak turbulence frame, several conversion mechanisms are presently debated to explain the EM emission. EM decay or coalescence $\left(L \rightarrow T_{f} \pm S\right)$, where a Langmuir wave $L$ decays into an EM wave $T_{f}$ close to the plasma frequency and an ion wave $S$, and linear mode conversion on density gradients (Schleyer et al., 2014and references therein) are the most advocated mechanisms to explain the emission at $T_{f}$. Regarding the emission at the harmonic, three main processes are proposed which all involve the presence of a current at $2 f_{p}$ in the plasma: (i) a two-step process involving Langmuir decay instability (LDI; Kruer, 1988) also called Langmuir electrostatic decay, followed by a coupling of electrostatic waves;

$$
L \rightarrow L^{\prime}+S \quad L^{\prime}+L \rightarrow T_{2 f}
$$


(ii) antenna mechanism based on the direct EM radiation by the second-order longitudinal current of electron density perturbations (Malaspina et al., 2010); and (iii) sheath rectification at the spacecraft (Graham et al., 2014).

Both processes (i) and (ii) are present in our numerical study, although we focus on the first one, based on the decay and coupling of waves, for which several proofs of occurrence in the interplanetary medium have been recently provided (Briand et al., 2014; Henri et al., 2009, 2010a).

Ginzburg and Zhelezniakov (1958) formulated the fundamental concept, which has been since then largely studied by numerous teams. However, few EM-kinetic simulations of the full problem (from electron beam to EM wave emission) have been performed. For example, Kasaba et al. (2001), Rhee et al. (2009), or Umeda (2010) performed 2-D particle-in-cell (PIC) simulations to study different aspects of the generation of the fundamental and harmonic EM radiation from a beam-plasma interaction. These simulations successfully showed the generation of the second harmonic $\left(T_{2 f}\right)$ from wave-wave interaction and gave estimate of the efficiency of the process and the directivity of the radiation. However, they suffer important limitations mostly due to the reduced number of particles per cell and a relatively small box limiting the number of EM wavelengths available in the simulation domain. In addition, only rather high-velocity $(0.5 c)$ and dense ( $1 \%$ to $10 \%$ of the background) electron beams have been considered. Recently, Thurgood and Tsiklauri (2015) partially overcame all these limitations by performing 2D3V PIC simulations with lower-density and slower beams and a larger number of particles per cell. Another approach of the problem has also been developed by Ziebell et al. (2015) who performed numerical simulations integrating the full set of weak turbulence equations.

In this paper we present a series of large-scale two-dimensional EM PIC simulations describing the nonlinear evolution of a suprathermal electron beam until the generation of the EM waves associated to solar radio emissions. A parametric study is performed to study the dependance of the radio emission properties as a function of the electron beam characteristics, in particular density and velocity. For this purpose, we have developed a 2D3V PIC model that uses a rather large number of particles per cell in order to reduce the noise level. This enables, on the one hand, to get rid of the spurious development of waves or of spurious scattering off macroparticles associated to the usual high noise level of PIC simulations and, on the other hand, to access lower-density beams. The model also considers a large box size, able to contain several EM wavelengths, in order to provide a fine sampling of the small EM waves wave vector. This is particularly necessary for the study of the intrinsic directivity of the emission process and its dependence on the electron beam properties. From these self-consistent numerical simulations, we report a parametric study of (i) the global efficiency of the conversion mechanism from the electron beam to the radio waves and (ii) the strong dependence of the radio wave directivity on the electron beam parameters, of key importance to infer accelerated electron beam properties from remote radio observations.

After a presentation of the numerical model (section 2), the simulations of solar radio waves generation are presented in section 3. In section 4, we discuss the numerical results and we explain the directivity diagram. Finally, we summarize our results in section 5.

\section{Numerical Model}

The generation of EM radiation starting from the evolution of a suprathermal electron beam is a multidimensional and multiscale physical problem which spans from the Debye length to several EM wavelengths. We model the solar wind plasma by considering the self-consistent kinetic evolution of a collisionless plasma, composed of electrons and ions, with the EM field through the Vlasov-Maxwell system of equations. These equations are solved numerically, using the PIC open source code "piccante" (Sgattoni et al., 2015, 2013). The simulations presented here are run in so-called 2.5-D (or 2D3V) geometry, that is, considering two spatial dimensions (2-D), using a Cartesian geometry, while the macroparticles have three velocity components $(3 \mathrm{~V})$. The simulation setup and parameters are summarized in the following, while details are given in Appendix A.

\subsection{Physical Parameters}

A plasma composed of electrons and protons fills the simulation box and forms the background plasma of the simulation. A uniform beam of suprathermal electrons fills the whole box and propagates with a velocity $v_{b}$ along the $x$ axis (hereafter defined as the direction parallel to the beam velocity, while the $y$ direction 
Table 1

Physical Parameters of the Simulations

\begin{tabular}{|c|c|c|c|c|}
\hline Plasma & & e-beam & & \\
\hline$T_{e}=4 \cdot 10^{-4} m_{e} c^{2}$ & & $T_{b}=T_{e}$ & & \\
\hline$T_{i}=T_{e} / 10$ & & $v_{b}=[9 ; 15] v_{t}$ & & \\
\hline$n_{0}=1$ & & $n_{b}=\left[5 \cdot 10^{-4}\right.$ to $\left.5 \cdot 10^{-3}\right] n_{0}$ & & \\
\hline$v_{b} \backslash n_{b}$ & $5 \cdot 10^{-4}$ & $1 \cdot 10^{-3}$ & $2 \cdot 10^{-3}$ & $5 \cdot 10^{-3}$ \\
\hline $15 v_{t}(0.3 c)$ & Run 1 & Run 2 & Run 3 & Run 4 \\
\hline $9 v_{t}(0.18 c)$ & Run 5 & Run 6 & Run 7 & Run 8 \\
\hline
\end{tabular}

is referred to as the perpendicular direction). The electron beam current is compensated by an opposite electron current carried by the background plasma. This ensures that at time $t=0$ the total current is correctly set to 0 . The global charge neutrality is imposed. Densities are normalized to the background proton plasma density $n_{0}=1$. A physical $m_{p} / m_{e}=1,836$ mass ratio is used. Both the electrons and protons of the background plasma have a Maxwellian velocity distribution. As the solar wind plasma is weakly magnetized (i.e., characterized by a larger-scale separation with an electron cyclotron frequency $\Omega_{c e} \simeq$ few tens of hertz, much smaller than the plasma frequency $\omega_{p} \simeq$ few tens fo kilohertz in the solar wind at 1 AU), the electrostatic waves such as the Langmuir waves are not affected by the electron gyromotion. Therefore, we hereafter consider the limit of a plasma initially unmagnetized, over which magnetic fluctuations can grow self-consistently. Because of this large scale-separation, the Langmuir wave is not affected by the electron gyromotion.

In the simulations discussed in this paper, the electron temperature is $T_{e}=4 \cdot 10^{-4} m_{e} c^{2}, \sim 200 \mathrm{eV} \sim 2 \cdot 10^{6} \mathrm{~K}$, so that the electron thermal velocity-to-speed of light ratio is $v_{t} / c=\sqrt{T_{e} / m_{e} c^{2}}=0.02$. It corresponds to electron temperatures expected in the solar wind close to the Sun, as should be measured by the next generations of solar space missions, Parker Solar Probe and Solar Orbiter, as well as BepiColombo when in the solar wind. The choice to work with these solar wind parameters also enables to mitigate the computational cost of the simulations. Indeed, the ratio between the larger (EM wavelength $\left.\lambda_{T_{2 f}}\right)$ and the smaller $\left(\lambda_{D}\right)$ length scales to be resolved by the code remains below 3 orders of magnitude. Since the actual solar wind ion-to-electron temperature ratio is highly variable but generally less than unity, we choose $T_{i} / T_{e}=0.1$, corresponding to what is observed in the slow solar wind (Montgomery et al., 1968; Robinson et al., 1993, e.g.). This regime enables the development and propagation of ion acoustic waves. The electron beam is modelled by a drifting Maxwellian velocity distribution with temperature $T_{b}=T_{e}$, that is, a beam thermal velocity $\Delta v_{b}$ equal to the thermal velocity of the bulk electron. In order to explore different beam conditions and enable parametric studies, the other characteristic parameters of the electron beam are varied as follows: The beam-to-total density is in the range $5 \cdot 10^{-4}<n_{b}<5 \cdot 10^{-3}$. These rather large values of beam density compared to the $10^{-6}$ usually observed in the interplanetary medium are necessary to increase the growth rate of the beam-plasma instability and reduce the computation time. However, they are still low compared to the former works cited in section 1 . Two values of the beam velocity have been considered: $v_{b}=9 v_{t}$ and $v_{b}=15 v_{t}$, corresponding, respectively, to $0.18 c$ and $0.3 c$ for the electron temperature used in these simulations, corresponding to the electron beam velocities typically observed with Type III radio bursts. A total of eight simulations have been performed varying the electron beam parameters, plus an extra one with

Table 2

Growth Rate $\gamma_{b p}$ and Regime (Fluid or Kinetic) for the Bump-on-Tail Instability of Each Run

\begin{tabular}{lcccccccc}
\hline Run \# & Run 1 & Run 2 & Run 3 & Run 4 & Run 5 & Run 6 & Run 7 & Run 8 \\
\hline$\left(\frac{n_{b}}{n_{0}}\right)^{1 / 3}$ & 0.08 & 0.10 & 0.13 & 0.17 & 0.08 & 0.10 & 0.13 & 0.17 \\
$\frac{\Delta v_{b}}{v_{b}}$ & 0.07 & 0.07 & 0.07 & 0.07 & 0.11 & 0.11 & 0.11 & 0.11 \\
$\frac{\gamma_{b p}}{\omega_{p}}$ & 0.055 & 0.069 & 0.087 & 0.12 & 0.015 & 0.031 & 0.087 & 0.12 \\
Limit & Fluid & Fluid & Fluid & Fluid & Kinetic & Kinetic/Fluid & Fluid & Fluid \\
\hline
\end{tabular}

Note. Runs 1-4 correspond to $v_{b}=15 v_{t e}$, Runs 5-8 to $v_{b}=9 v_{t e}$. 

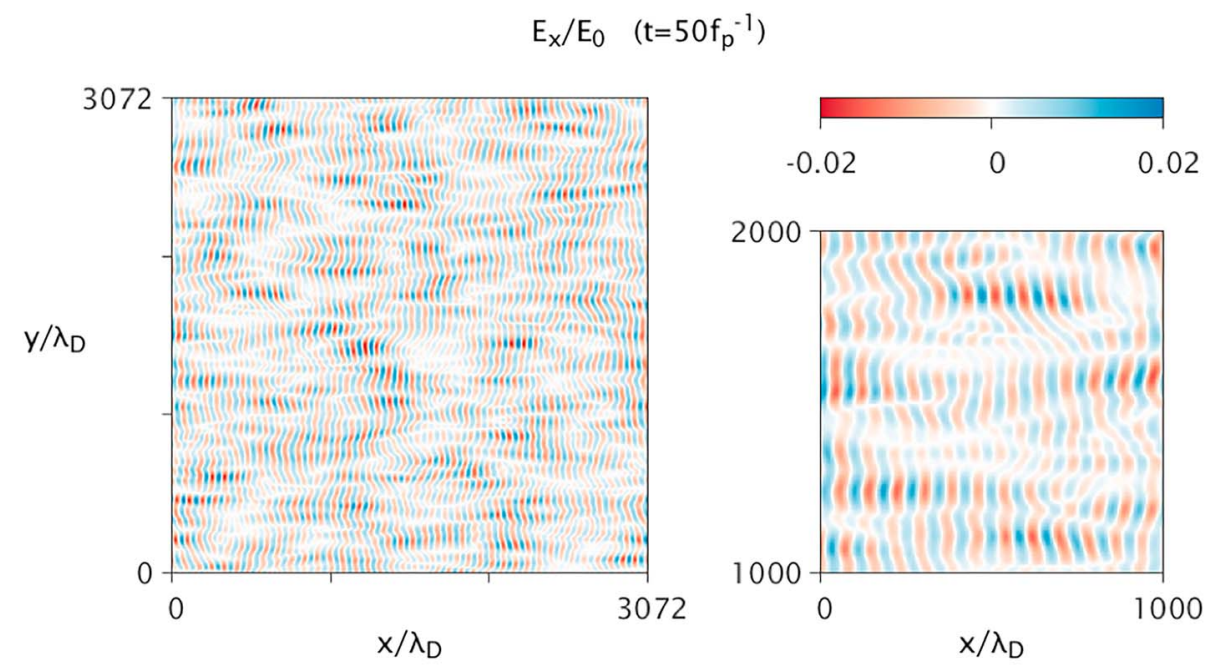

$E_{x} / E_{0} \quad\left(t=200 f_{p}^{-1}\right)$
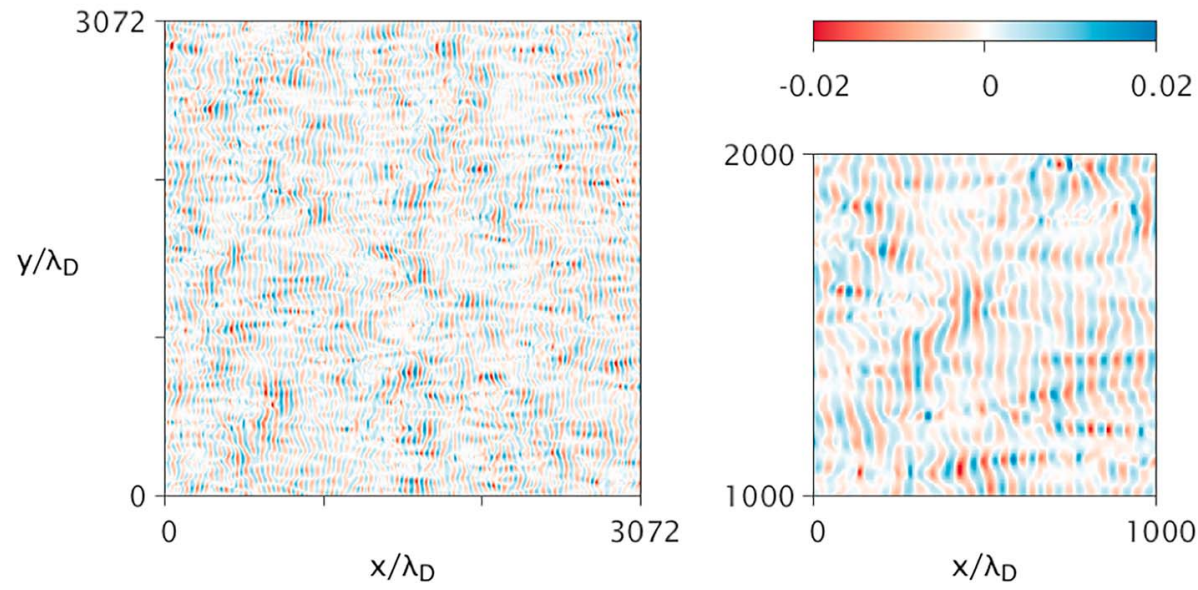

Figure 1. Snapshot of the parallel electric field, $E_{x}$, for Run 3 at time $t=50 f_{p}^{-1}$ (top panels) and at $t=200 f_{p}^{-1}$ (bottom panels). On the left column the full simulation box is presented, while the right column displays a small subset of the full box. The structures correspond to the electric field of Langmuir waves propagating (mostly) parallel to the beam propagation direction.

$v_{b}=7 v_{t}$ to extend the discussion of our parametric study. The plasma and electron beam parameters are summarized in Table 1, while Table 2 displays the growth rate of the bump-on-tail instability and kinetic or fluid regime of each simulation. The kinetic regime is reached when $\left(\frac{n_{b}}{n_{0}}\right)^{1 / 3} \ll \frac{\Delta v_{b}}{v_{b}}$. In the fluid regime the growth rate is given by: $\gamma_{b p}^{f l} / \omega_{p}=\sqrt{3} / 2 \times\left(n_{b} / 2 n_{0}\right)^{1 / 3}$ and is independent of the beam velocity. In the kinetic regime: $\gamma_{b p}^{k i n} / \omega_{p}=\sqrt{\pi / 8 e} \times\left(n_{b} / n_{0}\right)\left(v_{b} / \Delta v_{b}\right)^{2}$. If the simulation with the larger velocity are all clearly fluid, the simulations with the lower velocity are marginally fluid and more clearly kinetic for Run 5 .

\subsection{Numerical Setup}

The simulation box needs both (i) a small enough size of the grid cell, to ensure numerical stability, and (ii) a large enough grid size to contain several wavelengths of the EM radiation, to provide a good discretization of wave vector space and therefore ensure a thorough study of the EM emission directivity and of the conversion efficiency. With the parameters considered in this study $\left(v_{t}=0.02 c\right)$, the wave vector of the $T_{2 f}$ radiation is $k_{T_{2 f}} \lambda_{D}=\sqrt{3} v_{t} / c$, so that the EM wavelength is $\lambda_{T_{2 f}} \simeq 181 \lambda_{D}$. The simulation is therefore computed using (i) square cells with size $\Delta x=\Delta y=3 \lambda_{D}$, for which spurious numerical heating inherent to PIC codes is avoided (see Appendix A2), and (ii) on a square box size set to $L_{x}=L_{y}=3,072 \lambda_{D} \sim 17 \lambda_{T_{2 f}}$ and discretized in $1,024 \times 1,024$ cells, that ensures a satisfying discretization of the $T_{2 f}$ radiation Fourier modes (see Appendix A3). 


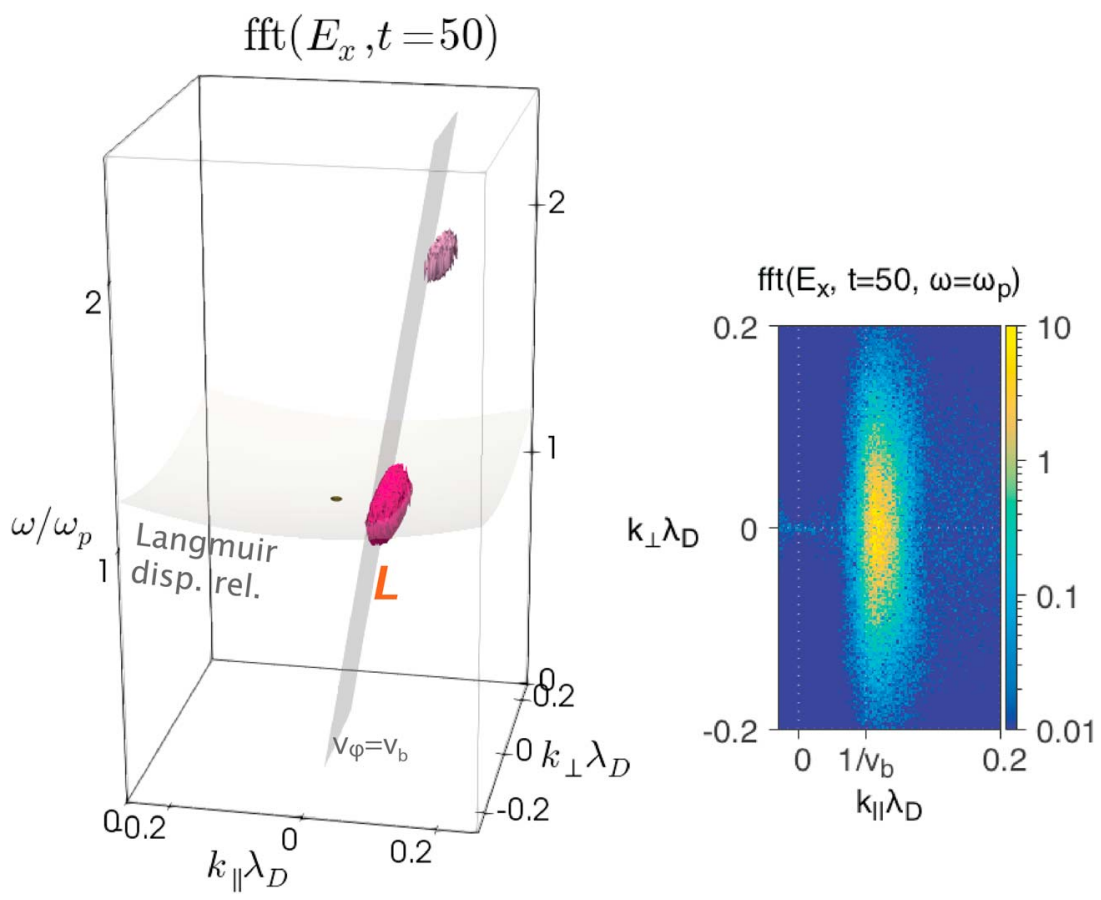

Figure 2. Spatiotemporal (2-D plus time) fast Fourier transform (left) and the $\omega=\omega_{p}$ slice (right) of the parallel electric field $\left(E_{x}\right)$ over a time window centered on $t=50 f_{p}^{-1}$ (Run 3). Two isosurfaces are drawn considering two different values of $\tilde{E}=\mathrm{fft} \quad\left(E_{x}\right)$ to highlight the two signals: at $\omega=\omega_{p}(L) \tilde{E}=0.25$ and at its harmonic $\omega=2 \omega_{p} \tilde{E}=0.05$. The gray almost horizontal paraboloid displays the dispersion relation of the Langmuir waves, while the inclined plane highlights the condition phase velocity equal to $v_{b}$.

The boundary conditions are periodic in both directions, reflecting the fact that the electron beam is much larger than the characteristic scale length of all the waves generation mechanisms. Both the beam and plasma background are initially uniform in space; that is, no large-scale density gradient is introduced. Small-scale density fluctuations are however generated self-consistently during the simulation.

The time step is set in order to ensure the Courant conditions $\Delta t=0.98 \Delta x /(\sqrt{2} c)$ and therefore numerical stability, since the code solves Maxwell's equations. In the following, the EM fields are normalized to $E_{0}=$ $m_{e} c \omega_{p} / e$.

The distribution function of the electrons of the background plasma and of the electron beam were sampled using 3,600 and 900 macroparticles per cell, respectively, while the background ion distribution function was sampled by 900 macroparticles per cell. This choice enables to reduce the intrinsic noise of PIC simulations, even in case of low density electron beams.

\section{Simulation Results: From a Suprathermal Electron Beam to Radio Emissions}

After describing the EM wave generation, the conversion efficiency from the suprathermal electron beam to the radio emissions and the radiation diagram are extracted from the simulations.

\subsection{Wave Generation}

3.1.1. Electrostatic Stage

At the beginning of the simulation, the electron beam-plasma instability generates primary Langmuir waves $(L)$ that propagate in the forward direction (with a phase velocity $v_{\phi, x}>0$ ). The parallel component of their phase velocity is expected to be close to the electron beam velocity $v_{\phi, x} \lesssim v_{b}$. The electron density fluctuations and the electric field, initially at 0 , grow as the beam-plasma instability takes place. One interesting feature is the self-consistent development of localized wave packets elongated in the parallel direction $x$ (see Figure 1 at $t=50 f_{p}^{-1}$ ), similar to what Graham et al. (2013) recently refers to as "Langmuir snakes." These structures have a typical length (in the direction parallel to the beam velocity) of $\ell_{x} \sim 8-10 \lambda_{L}$ and width (direction perpendicular to the beam velocity) $\ell_{y} \lesssim 1 \lambda_{L}$. 

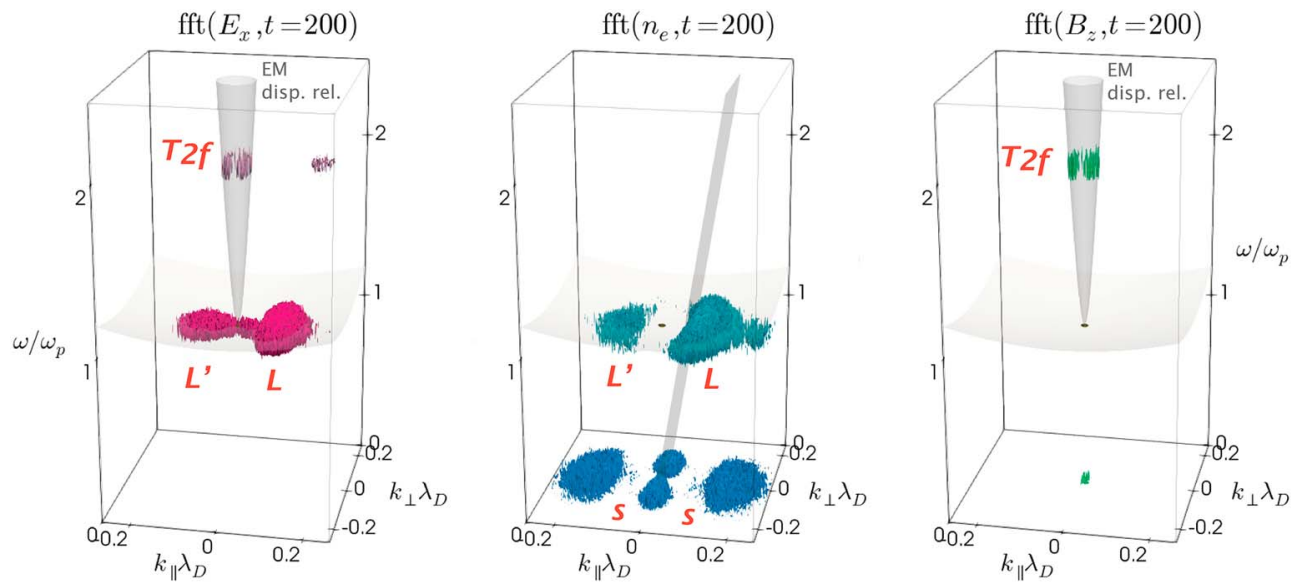

Figure 3. The $\mathbf{k}-\omega$ diagram around time $t=200 f_{p}^{-1}$ of the parallel electric field ( $E_{x}$, left), the electron density $\left(n_{e}\right.$, center), and the magnetic field ( $B_{z}$, right). Isosurfaces of the fast Fourier transform values are plotted selecting different values to highlight the different signals: (i) left panel: $L, L^{\prime}$ at $\omega=\omega_{p}$ for $\operatorname{fft}\left(E_{x}\right)=0.25$ and the electromagnetic signal at its harmonic $\omega=2 \omega_{p}$ for fft $\left(E_{x}\right)=0.05$; (ii) middle panel: $L, L^{\prime}$ at $\omega=\omega_{p}$ and the low-frequency signal in the electron density associated with acoustic waves $S$ and purely transverse modes are shown with isocontours for $\mathrm{fft}\left(n_{e}\right)=0.25$ and 0.5 , respectively. The narrow cone represents the dispersion relation of the electromagnetic waves.

At later stage, Langmuir wave packets characterized by smaller wavelengths and a lower level of coherency propagate in the plasma (as shown in Figure 1, bottom panels). It corresponds to the nonlinear stage of the electron beam evolution, associated to quasi-linear relaxation, when the electron velocity distribution function becomes unstable at smaller velocities, and is therefore responsible for the generation of Langmuir waves at smaller wavelengths. This is explained in further detail in Sgattoni et al. (2017).

To gain a better insight into the nature of the wave activity and its development during the simulations, spatiotemporal Fourier analysis of the fields and particle densities are performed considering several successive temporal windows of extension $L_{t}=20 f_{P}^{-1}$. This enables us to identify the nature of the waves by comparison with the known dispersion relation, to identify their direction of propagation, and finally to evaluate the energy content of each specific mode. Further details on this Fourier analysis can be found in Appendix A.

The Fourier transform of the electric field (and similarly of the electron density) confirms the excitation of the electrostatic Langmuir waves with the expected phase velocity, that is, $k_{L} \sim \omega_{p} / v_{b}$. This is illustrated for Run 3 in Figure 2, left panel. Note also the presence of the second harmonic of the electrostatic waves $L$. The generation of the harmonics of the electrostatic Langmuir wave has been extensively studied in previous works, in particular as nonlinear eigenmodes of a turbulent plasma associated with finite amplitude waves and in the framework of a nonlinear trapping theory (Klimas, 1983, 1990; Yoon et al., 2003). The amplitude of these perturbations is much lower than for the fundamental (the isocontour for the harmonic is 0.05 vs. 0.2 for the fundamental). The spectrum of the waves in the transverse direction is shown in Figure 2 (right) where the $\mathbf{k}$ space at $\omega=\omega_{p}$ is displayed. The Langmuir spectrum appears very large in the transverse direction with waves as far as $\pm 45^{\circ}$. In real space this results in narrow wave packets as seen in Figure 1 (top panels). The Langmuir waves excited by the instability can thus have a very large k aperture angle, and this aspect is essential to explain the generation and the angular spectrum of the EM emission at $T_{2 f}$. This is further detailed in section 4.2 .

At even later stage (e.g., $t=100-200 f_{p}^{-1}$ ), secondary Langmuir waves $L^{\prime}$ propagating backward develop (Figure 3 for Run 3). Low-frequency sound waves are found in both the electron and ion (not shown here) density plots in Fourier space; the sound waves spectra have maxima at $k_{S} \sim 2 k_{L}$. These structures are in agreement with what is expected from the electrostatic decay of the primary waves leading to the generation of secondary Langmuir waves $\left(L^{\prime}\right)$ and sound waves $(S)$.

In the Fourier transform of the electron density shown in Figure 3 (middle panel) a low-frequency (quasi-neutral) feature at $k_{x} \sim 0$ is also observed. We identify this as due to nonresonant beating of Langmuir waves, in the transverse direction, in analogy with what observed Riconda et al. (2011); Weber et al. (2012); Weber and Riconda (2015) in the context of the so-called two-plasmon decay (Kruer, 1988), the inverse 

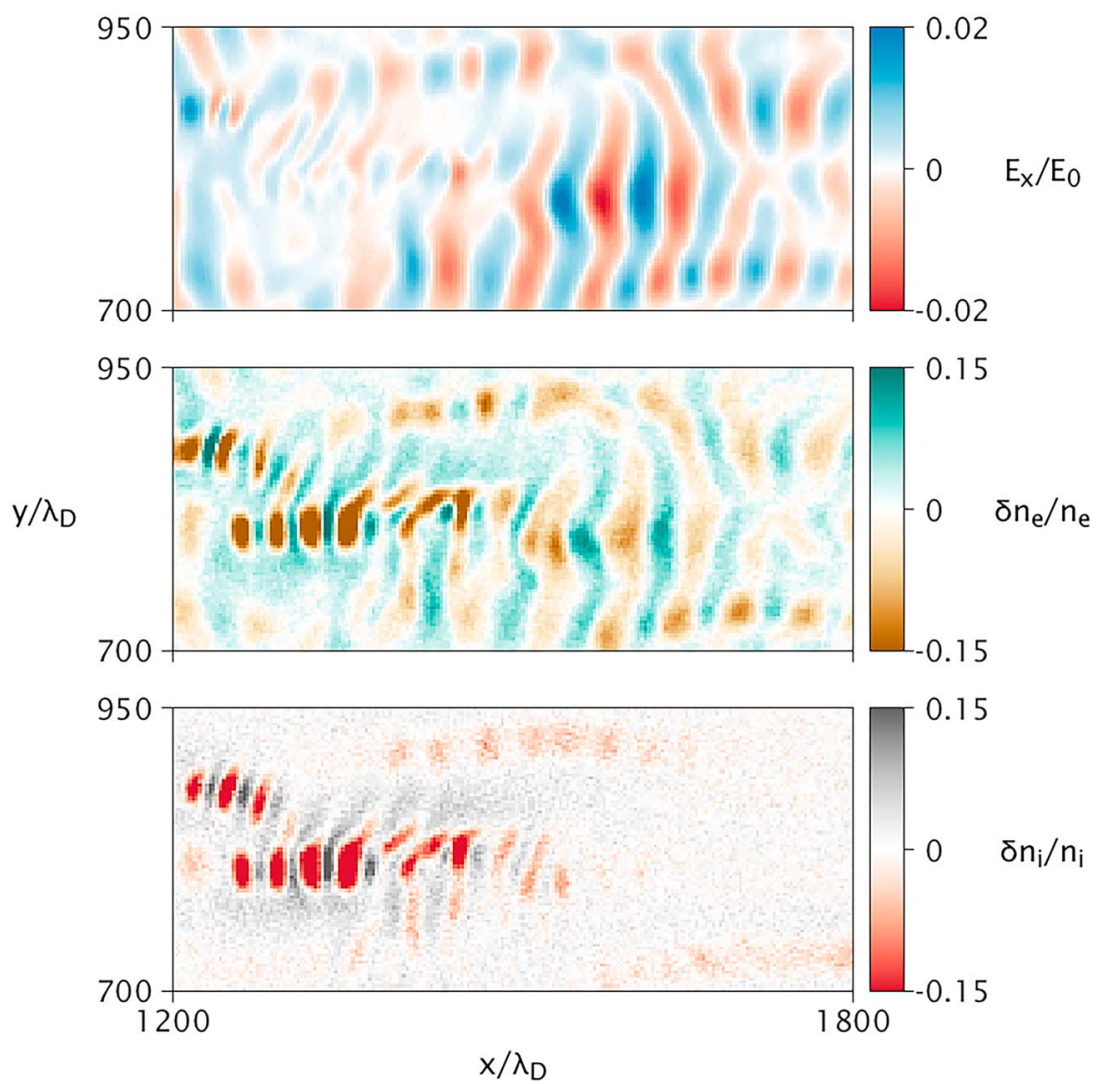

Figure 4. Enlargements of the simulation box of Run 3 showing (from top to bottom) the parallel electric field $\left(E_{x}\right)$ and electron and ion density perturbations $\left(\delta n_{e} / n_{e}\right.$ and $\left.\delta n_{i} / n_{i}\right)$ at $t=200 f_{p}^{-1}$. The bright structures on the two bottom panels evidence ion acoustic waves.

process of the $T_{2 f}$ generation, that is, $T_{2 f} \rightarrow L+L^{\prime}$. As in the case of the Langmuir waves produced by the two-plasmon decay, these transverse modes contribute to creating elongated structures in real space in the direction parallel to the electron beam direction, even in the weak turbulence regime.

A zoom of the electrostatic field and of the electron and ion densities is shown in Figure 4: The electric field (top panel) is a tracer for the Langmuir waves, and the ion density fluctuations (bottom panel) is a tracer for the ion acoustic wave packets, while the electron density fluctuations (middle panel) traces both electrostatic waves. The localized wave packets in the ion density (bottom panel), with typical width of a few Langmuir wavelengths along $x$ and of a few Langmuir wavelengths in the perpendicular direction, are therefore the ion acoustic waves generated by the Langmuir decay.

\subsubsection{EM Emission}

As soon as the secondary Langmuir waves are excited, the presence at the same time of $L$ and $L^{\prime}$ waves lead to the EM harmonic emission. These EM waves are identified by the magnetic field $B_{z}$ (right panel of Figure 3) orthogonal to the simulation plane $(x-y)$ and the two components of the electric field $E_{x}$ (left panel of Figure 3) and $E_{y}$ (not shown). The Fourier analysis of the fields confirms the presence of a strong emission at $\omega=2 \omega_{p}$ laying on the dispersion relation of the EM waves.

To further study the link between the two families of electrostatic waves (backward and forward) and the generation of the EM waves, the time evolution of the normalized energy carried by each wave is presented in Figure 5. The mother Langmuir waves $L$ first grow, followed by the increase of the daughter Langmuir waves $L^{\prime}$. The growth of the EM radiation at $2 f_{p}$ starts later in time and follows the growth of the daughter waves rather than the growth of the mother waves. The relationship between $L^{\prime}$ and $T_{2 f}$ is even clearer for an electron beam at low velocity (Figure 5, right). It is important to stress that the final energy in the EM waves is about few $10^{-5}$ of the beam kinetic energy, irrespective of the initial beam velocity. Note that this value is obtained from the Fourier analysis, thus integrated over all emission angles. 


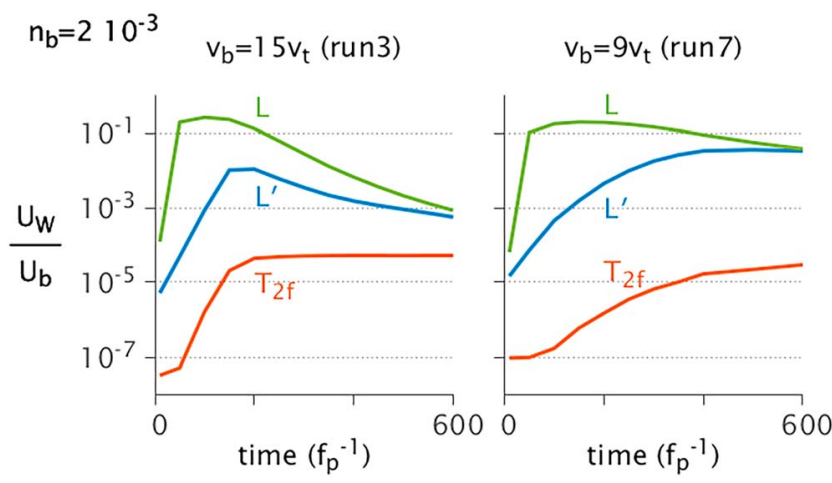

Figure 5. Time evolution of the wave energy $\left(U_{\mathrm{w}}\right.$ relative to the initial beam kinetic energy $U_{\mathrm{b}}$ ) distinguishing between forward and backward propagating Langmuir waves, $L$ and $L^{\prime}$, respectively, and electromagnetic emission at $2 \omega_{p}, T_{2 f}$, for two beam velocities; (left) Run 3 and (right) Run 7.
In summary, our PIC simulations reproduce the development of the waves resulting from the relaxation of an electron beam propagating in a uniform plasma, from the electrostatic stage up to the development of the second harmonic of the EM radiation. The emission of an EM radiation at $2 f_{p}$ is compatible with a two-step process: (i) the decay of large-amplitude Langmuir waves (step 2 in Figure 6), followed by (ii) the coupling of two Langmuir waves into an EM wave at twice the plasma frequency (step 3 in Figure 6). In the rest of this study, we focus on the $2 f_{p}$ EM radiation. Regarding the emission of an EM radiation at $f_{p}$ (step 4 in Figure 6), it is hardly discernible in our simulations. First, a much larger box size should be used to properly measure the very long wavelength of the fundamental emission. Second, regarding the generation of the fundamental EM emission itself, it is worth recalling that two competitive processes can play a role: the Langmuir wave decay into an ion acoustic wave and the transverse radiation or the Langmuir wave scattering off thermal ions to generate the transverse radiation, which were found to be equally important, at least in the framework of weak turbulence (Ziebell et al., 2015). Future large-scale, kinetic studies specifically dedicated to identifying the mechanism responsible for the generation of fundamental EM emissions should therefore, first, include a density gradient for the radiation to exit the plasma and, second, take into account the fact that the discretization of the distribution function with macroparticles, as it is done in PIC simulations, tends to increase spuriously the thermal noise in the plasma, which may in turn favor the process of wave scattering off macroparticles.

\subsection{Conversion Efficiency From the Electron Beam to the Radio Emission}

While the radio emissions associated to $T_{2 f}$ enable to follow the propagation of suprathermal electron beams in the solar wind and upstream quasi-parallel collisionless shocks, they have given little insight so far regarding the efficiency of the energy transfer from the electron beam to the EM radiation. Robinson and Cairns (1998) provided a theoretical expression for the efficiency of the energy conversion; Umeda (2010) performed some estimation of the efficiency but for rather dense and fast electron beams. Our simulations enable to address this issue by following the time evolution of the EM radiation and, for the first time, for a variety of beam velocities and densities.

Interestingly, for all the cases considered in our parametric study, we report that about few $10^{-5}$ of the total beam (kinetic) energy is transferred into EM radiation at $2 f_{p}$ in all directions. This is illustrated in Figure 7, which displays the time evolution of the radio wave energy (right panel), relative to the initial electron beam (kinetic) energy, for the different electron beam densities considered in our parametric study, ranging over an order of magnitude. It complements Figure 5, which displays the time evolution of the radio wave energy (in red) for two different beam velocities and the same beam density, also saturation at about $10^{-5}$ of the total beam (kinetic) energy.

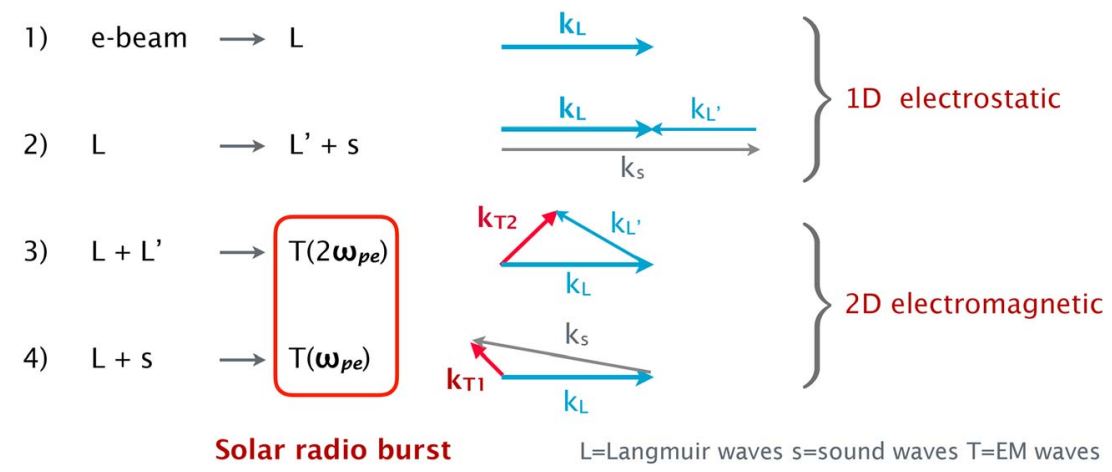

Figure 6. Scheme of the multistep process of the radio emission: (1) Bump-on-tail instability leads to Langmuir waves $(L),(2)$ electrostatic decay of $(L)$ into secondary Langmuir waves $\left(L^{\prime}\right)$ and ion acoustic waves $(S)$, (3) two-plasmon $\left(L+L^{\prime}\right)$ coalescence to generate electromagnetic waves at $2 \omega_{p}\left(T_{2}\right)$, and (4) plasmon-sound wave interaction or plasmon scattering off thermal ions to generate electromagnetic waves at $\omega_{p}\left(T_{1}\right)$. 


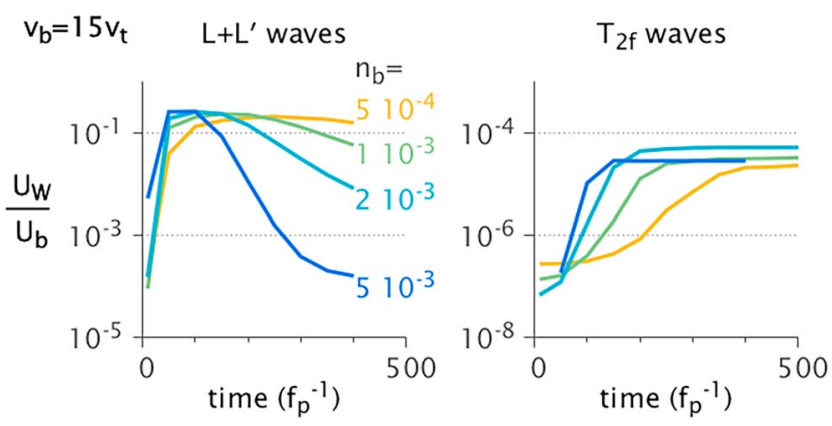

Figure 7. Time evolution of the total energy of the Langmuir waves (left) and electromagnetic waves at $\omega=2 \omega_{p}$ (right) with respect to the initial total kinetic energy of the electron beam (Runs 1 to 4).

In the following, we concentrate on the analysis of the radiation pattern of the EM waves at $2 f_{p}$.

\subsection{Angular Distribution of EM Emission}

The theoretical results on the expected directivity of the coupling processes are far from being conclusive: Melrose (1973) predicted a preferential forward emission, and Takakura (1979) suggested that both forward and backward emissions are possible. Observations do not appear to be more conclusive. Fitzenreiter et al. (1976) concluded from a statistical analysis of IPM-6 data that the emission is almost symmetric with respect to the magnetic field with a radiation aperture of about $35^{\circ}$. However, Hoang et al. (1997) and Bonnin et al. (2008), analyzing observations from space radio instruments, showed that the EM emissions at low frequency display a pronounced drift toward the east compared to the direction of the magnetic field (i.e., the direction of propagation of the bulk of the electrons ejected from the Sun during an eruption). Several works have also tackled this issue of radiation directivity from 2-D EM simulations. Rhee et al. (2009) and Umeda (2010) from PIC simulations and Ziebell et al. (2014) from the resolution of the weak turbulence equations have found a privileged direction between $30^{\circ}$ and $60^{\circ}$ in the forward direction and $130^{\circ}$ and $160^{\circ}$ in the backward direction, and an increase of the directivity with the intensity of the EM emission. Also, Figure 4(b) of Rhee et al. (2009) displays a clear backward (and almost no forward) emission using a very large beam velocity of $0.5 c$.

The large size of our numerical box enables to better resolve the angular distribution of EM emissions compared to former works (see Appendix A and Figure A1 therein for a detailed discussion on the choice of the numerical parameters). The integral of the electric field power spectrum is computed over angle bins of $15^{\circ}$. Figure 8 displays the angular distribution of the EM wave on the simulation plane containing the beam propagation direction. On the one hand, we confirm former results that have shown that two privileged directions of radiation emission are observed: at $40 \pm 10^{\circ}$ (i.e., forward, which corresponds to an anti-Sunward direction) and $150 \pm 10^{\circ}$ (i.e., backward). On the other hand, we also show that, although the global (omnidirection) conversion efficiency was shown not to depend much on the electron beam parameters,
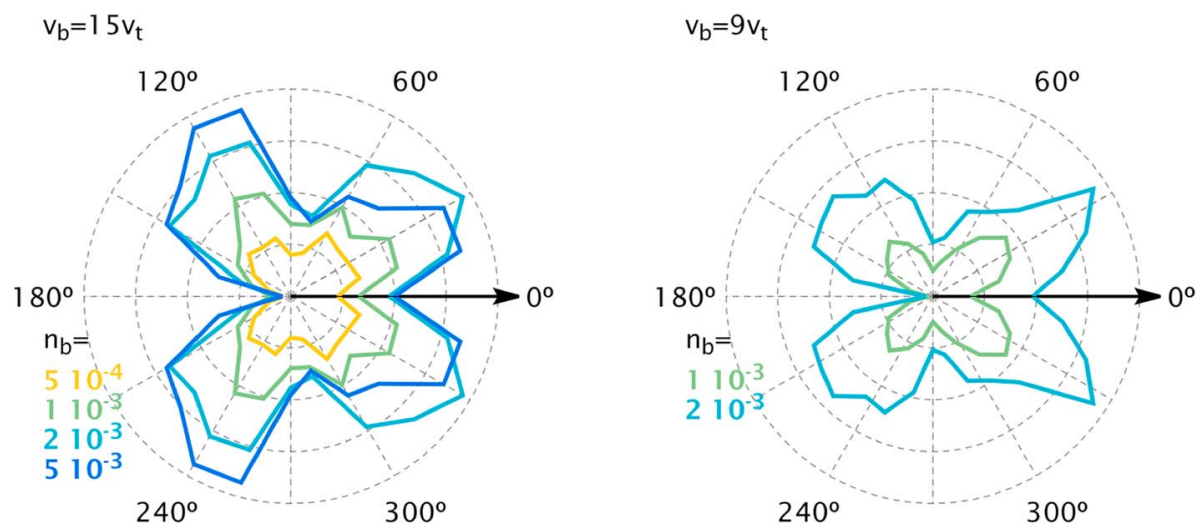

Figure 8. Polar diagram of the radio emission amplitude (linear scale) of the harmonic electromagnetic emission for two different electron beam velocities ( $15 v_{t h}$ on left panel and $9 v_{t h}$ on the right) and several beam densities, corresponding to Runs 1 to 4 . The $0^{\circ}$ indicates the direction of the electron beam (i.e., the forward direction). 
the directivity itself of the EM emission strongly depends on the electron beam properties. First, the directivity of the EM emission depends on the density of the electron beam: The denser the beam, the more pronounced the directivity. Also note the asymmetry of the emission along the beam direction: Backward emission is almost 0 whatever the density. Second, the directivity becomes more pronounced as the beam velocity decreases (compare left and right plots of Figure 8 for the same density). Third, the forward propagation is privileged for low-velocity beams. The reason for this dependence on the beam velocity is discussed below (section 4).

Previous observations report Eastward shifted radiation pattern at about $40^{\circ}$ and were interpreted as the result of the EM wave scattering in the interplanetary medium (Bonnin et al., 2008; Hoang et al., 1997; Poquérusse et al., 1996). However, conversely to these conclusions, our results suggest that the observed directivity can also originate from the harmonic radiation pattern of the coupling process itself, without requiring any scattering. Propagation effects can still affect the width of the radiation pattern, but this cannot be tested in our simulations which represent a very small space volume.

\section{Discussion}

\subsection{Efficiency}

Our evaluation of the global energy transfer efficiency from the electron beam to the EM emission confirms the previous evaluation of Umeda (2010) and the values that can be deduced from the figures of Rhee et al. (2009). They are however at least 3 orders of magnitude larger than the theoretical estimation in Robinson and Cairns (1998). This apparent discrepancy is due in part to the fact that the energy estimated in our paper is integrated over all emission angles. We observe that the omnidirectional, total energy transferred from the electron beam to the radio waves scales as $10^{-5}$ the kinetic energy of the electron beam, whatever the electron beam density and velocity, at least in the regime of parameters considered in this parametric study. The independence of the conversion efficiency versus the beam density and the beam velocity can be understood by the fact that the generation of the Langmuir waves is in all cases saturated. Therefore, we expect the global conversion efficiency, and therefore the energy deposited in the radio waves, to decrease in situations where the beam-plasma instability would not be saturated.

Previous studies have emphasized that the efficiency of the first step of the radio emission conversion mechanism (namely, the LDI) increases with the electron-to-ion temperature ratio (Henri et al., 2010b). We therefore expect that, although the conversion mechanism efficiency is shown not to depend much on the beam properties itself, it should somehow depend on some plasma bulk properties such as the electron-to-ion temperature ratio. This can be expected since the electrostatic waves are carried by the bulk plasma itself. Provided that the effects of electron beam itself and those of the background bulk plasma can be distinguished, it would open interesting applications, in particular on how to use solar wind radio emissions as a diagnostic for both (i) electron beam and (ii) the background plasma, and therefore eventually better characterize electron acceleration mechanisms from remote radio observations. In this study, we have chosen to concentrate our parametric study on the electron beam properties. A parametric study based on bulk plasma properties should undoubtedly be a complementary next step in our understanding of the conversion efficiency from accelerated suprathermal electrons to radio emissions.

Finally, even though the box size is significantly larger than in previous numerical works so that the directivity of the harmonic emission can be studied in detail, it is still too small to properly enable the development of the fundamental emission (though an hint of fundamental EM emission can be visible at $f_{p e}$ in Figure 3, right panel). This means that one channel for the energy transfer to the EM radiation is reduced. Thus, the efficiency obtained in our simulations regarding the harmonic may well be considered an upper limit value.

The fact that the energy transferred from the electron beam to the radio emission only depends on the electron beam total kinetic energy suggests that it may be possible to retrieve the free energy available locally (i.e., stored in electron beam kinetic energy) from the remotely observed radio emission. However, as the radio emission is observed at a given point only, one should also know the directivity properties of the radio emission in order to get back to the electron beam properties.

\subsection{Directivity}

In the case of radio emission at $T_{2 f}$ due to the coupling between a primary forward propagating Langmuir wave $L$ generated by the beam-plasma instability, and a second backward-propagating Langmuir wave $L^{\prime}$, possibly generated by $L D I$, two conditions have to be taken into account that will determine the amplitude 


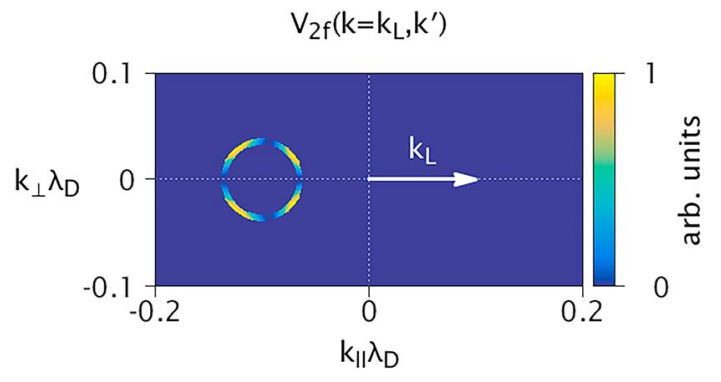

Figure 9. Coupling factor $V_{2 f}\left(\mathbf{k}=\mathbf{k}_{L}, \mathbf{k}^{\prime}\right)$, recalled in equation (5), as a function of the wave vector of the secondary Langmuir waves for a given primary Langmuir wave vector $k_{L}$. and the angular spectrum of the emission. First, energy and momentum conservation laws must be satisfied. In the case of the harmonic emission, they read as follows:

$$
\begin{aligned}
\overrightarrow{k_{L}}+\overrightarrow{k_{L^{\prime}}} & =\overrightarrow{k_{2 f}} \\
\omega_{L}+\omega_{L^{\prime}} & =\omega_{2 f}
\end{aligned}
$$

However, even if these conservation laws are satisfied, the source term corresponding to the emission at $T_{2 f}$ depends on the precise geometry of the coupling. Considering that, for an EM transverse wave, one has to consider only the component of the current that is perpendicular to the direction of emission, the source term writes

$$
\begin{gathered}
\partial_{t} E_{2 f} \equiv V_{2 f}\left(\mathbf{k}_{L}, \mathbf{k}_{L^{\prime}}, E_{L}, E_{L^{\prime}}\right) \\
\sim E_{L} E_{L^{\prime}} \frac{\omega_{L^{\prime}} k_{L^{\prime}}^{2}-\omega_{L} k_{L}^{2}}{k_{L} k_{L^{\prime}} k_{2 f}} \sin \theta
\end{gathered}
$$

where $\theta$ is the angle between the two Langmuir wave vectors of $E_{L}$ and $E_{L^{\prime}}$. The coupling is only possible if (i) the two Langmuir wave vectors have different lengths (otherwise, $\omega_{L}=\omega_{L^{\prime}}$ and the numerator in the source term in equation (5) vanishes) and (ii) form a nonzero angle $\theta$. This source term is shown in Figure 9 for a given wave vector $\vec{k}_{L}$ and different wave vectors $\overrightarrow{k_{L^{\prime}}}$. This plot evidences, at least for a single $\overrightarrow{k_{L}}$, the growth of a quadrupole-like angular pattern.

In practice, the primary as well as the secondary Langmuir waves have a wide angular spectrum and a large dispersion in lengths $k_{L}$ and $k_{L^{\prime}}$, as shown in Figure 10. The final angular spectrum of the radiation at $T_{2 f}$ is thus a convolution between the angular and wave vector spectrum of waves $L$ and $L^{\prime}$ and the coupling factor represented in Figure 9. As explained in the following, in most cases, this convolution leads to an emission in a very broad angular domain.

The combination of geometric consideration and of the Langmuir spectral width on the directivity of EM emissions are illustrated in Figures 11 and 12, for two situations corresponding to different spectra of the Langmuir waves. Both are obtained for the same beam density $n_{b} / n_{0}=2 \times 10^{-3}$. In both Figures, the red circle represents the modulus of the wave vector of the transverse wave $T_{2 f}$, which, in real units and for a frequency $2 f_{p}$ is close to $k_{2 f} \simeq \sqrt{3} \omega_{p} / c$. The direction of the emission corresponds to the direction of the wave vector $\overrightarrow{\mathbf{k}_{2 f}}$. In order to satisfy the conservation (equation (2)) the end of the wave vector $\overrightarrow{k_{L^{\prime}}}$ has to lay on the light blue circle, on the left side of the figures. The light blue circle therefore represents the part of the backscattered Langmuir wave spectrum available for conversion to EM emission for a single primary wave vector $\vec{k}_{L}$.

The LDI depends on the phase velocity of the primary waves, which is close to the beam velocity, and in particular, for the considered parameters, the smaller the velocity, the narrower the spectrum of the secondary Langmuir waves. These different kinds of spectra will lead to different coupling geometries and different directions for the EM waves. In a first case (Figure 11), the growth rate is quite large $\left(\gamma_{b p} / \omega_{p}=0.087\right)$ and $v_{b}=15 v_{t}$. In a second case (Figure 12), both the growth rate and the beam velocity are smaller $\left(\gamma_{b p} / \omega_{p}=0.037\right.$ and $\left.v_{b}=7 v_{t}\right)$, resulting in a narrower spectrum for the primary Langmuir waves.

Figure 11 shows that, for a beam velocity $v_{b}=15 v_{t}$, secondary Langmuir waves are found in all directions available for wave coupling (blue circle), so that both forward and backward EM emissions will be generated. The bottom panel also illustrates the possibility to emit transverse waves at $T_{2 f}$ along the axis of the electron beam itself, at least in the limit of an 

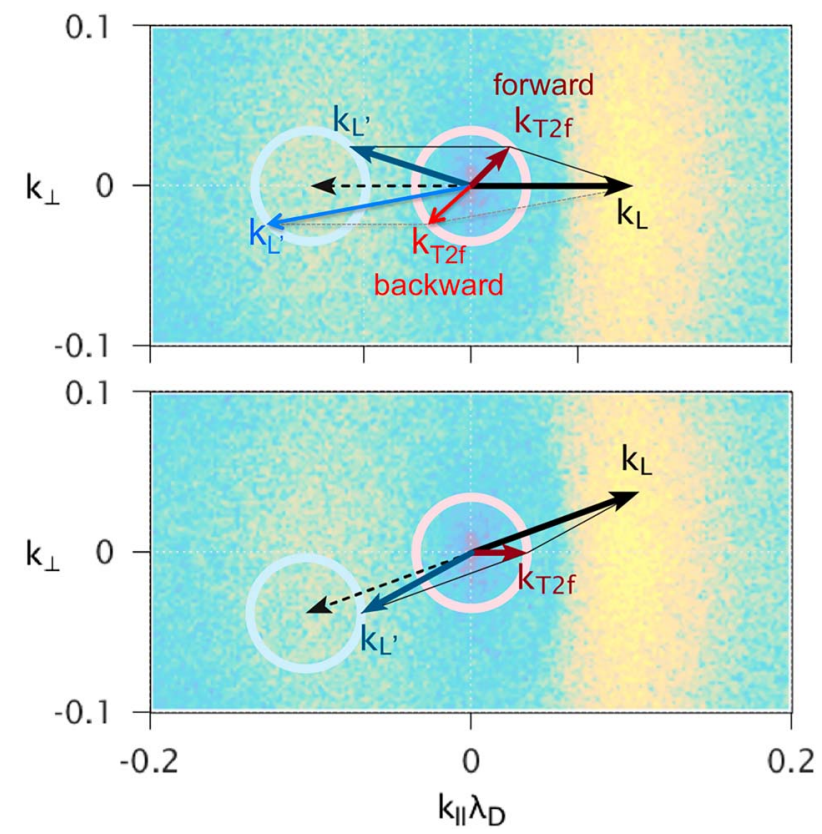

Figure 11. Different configurations of harmonic emission wave vector are superimposed on the Langmuir spectrum as in Figure 10. (top panel) The EM emission $\left(T_{2 f}\right)$ is at $45^{\circ}$ forward (upper half of the top panel) or backward (lower part of the top panel) for a same $k_{L}$ parallel to the beam velocity $v_{b}$. (bottom) The EM emission is at $0^{\circ}\left(\| v_{b}\right)$, and $k_{L}$ is at $20^{\circ}$. $\mathrm{EM}=$ electromagnetic. unmagnetized plasma. On the contrary, Figure 12 shows that for a lower beam velocity $v_{b}=7 v_{t}$, the primary waves have a much longer wave vector and LDI does not produce secondary Langmuir waves in all directions available for wave coupling (in particular not in the left side of the light blue circle), thus hindering the EM emission in the backward direction.

The above discussion explains the directivity of the emission at $T_{2 f}$ observed in our simulations, shown in Figure 8, and how they directly inherit from the electron beam properties. Namely, larger beam densities widen the Langmuir waves spectrum, which in turn leads to a larger available angular domain for the generation of the EM emission, including the backward half-plane and the 0deg on-axis direction. In addition, in agreement with Figure 9, the privileged angles of emission are close to $45^{\circ}$ and $135^{\circ}$. Instead, at lower beam densities or velocities, the spectrum of the primary and secondary Langmuir waves are narrower and the forward half-plane is privileged.

The properties of electron beams propagating in the heliosphere, in particular their density and velocity, depend on the electron acceleration mechanism, that can vary from electron accelerated during solar flares to electrons accelerated in front of shocks generated in the heliosphere (see a recent review paper on that aspect by Briand, 2015). The properties of the associated radio emission should reflect those of the electron beams. First, these results indicates that the radio emissions associated to electron beams characterized by smaller velocities shall show a more pronounced forward-directivity. Second, these results allow to extrapolate that in the regime of very small electron beam densities, such as those typically associated with solar flares (usually observed to be as low as $10^{-5}$ to $10^{-6}$ the solar wind plasma density), the quadrupolar directivity is expected to be less pronounced, while the forward directivity should strongly dominate the emission diagram (see Figure 8).

Third, we recall that the simulations were performed without an initial background external magnetic field. This unmagnetized limit is justified by the fact that the solar wind is characterized by a large scale separation between the electron cyclotron frequency and the plasma frequency. However, the presence of a magnetic field may reduce the number of oblique Electrostatic (ES) modes (Timofeev, 2012) and could therefore even increase the directivity of the EM radiation of radio emissions in the heliosphere.

Finally, we emphasize that our conclusions in terms of expected directivity are valid in the radio emission source region. Away from the source region, the EM wave directivity has to be mitigated by the possible reflection and scattering of the radio emission in the inhomogeneous solar wind plasma. First, because of the plasma density decrease with the heliocentric distance, the backward radio emission directed toward the Sun, that is, toward increasing densities, will eventually be reflected forward and add up to the forward EM emission. Second, in a strongly

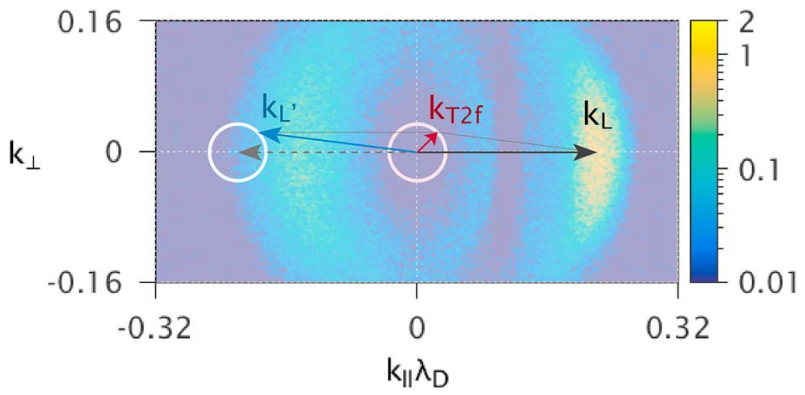

Figure 12. Similar to Figure 11 but considering a low beam velocity $v_{b}=7 v_{t}=0.14 c$ : superposition of the Langmuir waves spectra at time $t=600 f_{p}^{-1}$ with a sketch of a possible configuration of wave vectors coupling for the harmonic emission. inhomogeneous medium such as the solar wind, the radio emission may be scattered by over-dense plasma regions. This means that knowing the radiation diagram of the EM emission is not the end of the story. One should obviously also consider the propagation properties of radio waves in the inhomogeneous solar wind, in order to infer the properties of the electron beam at the emission source from remote radio observations and therefore reach the desired final goal: Use solar wind radio emissions as a diagnostic for electron acceleration mechanisms.

\subsection{Nonlinear Mode Conversion Versus Other Mechanisms}

To generate an EM wave at a given frequency, a current at the same frequency has to be present in the plasma. The emission at $T_{2 f}$ is thus due to the presence of electron currents at $2 f_{p}$. This current can either be related to the coupling between two different Langmuir waves in the form 
$-e\left(n_{L} v_{L^{\prime}}+n_{L^{\prime}} v_{L}\right)$, as discussed above for the backscattered waves generated by LDI but also valid for other backward propagating Langmuir waves, or the second harmonic of intense wave packets of Langmuir waves in the form $-e n_{L} v_{L}$.

First, in a realistic scenario considering an inhomogeneous plasma, two channels enable to generate backward propagating Langmuir waves $L^{\prime}$ : wave reflection on density gradients on scales much larger than the Langmuir wavelength (Krafft et al., 2015; Thurgood \& Tsiklauri, 2016) and electrostatic decay. Although density gradients are permitted in our simulations, large-scale density gradients are not included initially and do not develop. Therefore, backward propagating Langmuir waves cannot be generated through reflection on such density gradients. Instead, in our simulations, backward propagating Langmuir waves can only be produced through the Langmuir electrostatic decay. Therefore, in an inhomogeneous solar wind, or in solar wind regions characterized by a higher level of compressibility, we expect the coalescence of counterpropagating Langmuir wave packets to be even more efficient. In the case when backward propagating Langmuir waves are predominantly generated by reflection on larger scale density gradients, the previous discussion on the EM wave directivity in section 4.2 is still valid and may be adapted to the expected spectra of backward propagating reflected Langmuir waves $L^{\prime}$ to infer the EM wave directivity.

Second, the antenna mechanism (Malaspina et al., 2013), which relies on the nonlinear current at the second harmonic of the primary Langmuir waves, can also act as a source term for the generation of EM emissions. However, even if all the primary waves can in principle contribute, given that the frequency and wave vector of this second harmonic do not verify the dispersion relation of the transverse wave $T_{2 f}$, only a localized intense structure (ILS) will give nonzero emission (Malaspina et al., 2010). Mathematically, the emission is the integral over the whole volume of an ILS of the emission of each oscillating electron at $2 f_{p}$ taking into account the phase (i.e., the distance) between each emitter and the receiver. In our case, we have elongated structures, and the emitting region in the direction perpendicular to the direction of observation and perpendicular to the phase velocity of the Langmuir wave will be of the order of the EM wavelength, as for spherical ILS's (Malaspina et al., 2013). The emitting region in the direction perpendicular to the direction of observation but parallel to the phase velocity of the Langmuir waves will be instead of the order of the wavelength of the Langmuir wave. Thus, in order for this mechanism to be efficient, a very narrow structure is needed in the parallel direction, that is, a large Fourier spectrum of the Langmuir waves, such that $\delta k \sim 2 k_{L}$. This is not observed in our simulations: In Figure 1 bottom panels, we observe that the width of the structures is of the order of the EM wavelength at $2 f_{p}$ and their length is many times the Langmuir wavelength. As a result the efficiency of the Antenna mechanism is decreased in the case of our simulations.

\section{Conclusions}

This paper presents self-consistent 2D3V EM full PIC simulations of the relaxation of an electron beam in an initially homogeneous solar wind plasma. The questions tackled by the study are the following: Is there a privileged direction of emission from the fundamental physical process at the origin of the EM radiation? What is the efficiency of the conversion mechanism? Answering the first question enables to set some constraints on the discussion of the role of the scattering in the interplanetary medium, necessary to localize the emission sources. Answering the second question is fundamental to estimate the beam energy from remote observations of the EM radiation. It shall also enable to constrain other types of simulations, such as quasi-linear simulations.

The full kinetic simulations have allowed to follow in time the generation of different waves: the Langmuir waves resulting from the bump-on-tail instability, the Langmuir daughter waves and the ion sound waves resulting from the electrostatic decay of the mother Langmuir waves and, finally, the emergence of the harmonic EM waves at $2 f_{p}$. Thus, both the electrostatic and EM stages are well identified. However, the limited size of the simulation box and the absence of a large-scale density gradient likely impede the observation of the fundamental EM emission at $f_{p}$. These simulations lead to the following results:

1. The global (omnidirectional) conversion efficiency from the electron beam to the radio wave is shown to be very weakly dependent on the electron beam properties. A total of about $10^{-5}$ of the initial beam kinetic energy is observed to be transferred into EM waves at $2 f_{p}$, whatever the electron beam density (from $5 \cdot 10^{-4}$ to $5 \cdot 10^{-3} n_{0}$ ) and velocity ( 9 and $15 v_{t e}$ ) covered by our parametric study.

2. On the opposite, the radio wave directivity strongly depends on the electron beam parameters. It was demonstrated how it is strongly correlated to the electron beam instability through the properties of the 
generated Langmuir spectra (forward and backward through the ES decay). The EM radiation is emitted in privileged directions (cf. Figure 8): at about $45^{\circ}$ for forward propagating radiation and $-60^{\circ}$ for backward propagating radiation. The strictly backward $\left(180^{\circ}\right)$ propagation is completely absent. The radiation pattern is more pronounced for high-density beams. Moreover, a forward versus backward asymmetry of the EM radiation is shown for low-velocity electron beams.

Given the observed dependence of the radio emission directivity properties on the electron beams that are responsible for its generation, these new results are expected to have a direct impact on the understanding of the radio emission source properties that can most of the time be acquired only through radio remote measurements.

The future Parker Solar Probe, Solar Orbiter, and BepiColombo/MMO will likely cross the emission sources, reducing dramatically the role of the scattering, enabling thus to discriminate the effects of EM radiation scattering in the interplanetary medium from the directivity of the emission processes itself. Another way to check these conclusions is to perform laboratory experiments, in particular, on laser infrastructures. In our setup in preparation, a first laser will produce the plasma, while the decay of a second laser entering this plasma produces the Langmuir waves. The experiment will be performed on the LULI2000 laser facility.

\section{Appendix A: Numerical Setup}

The numerical setup is discussed in further detail in this appendix.

\section{A1. Return Current}

The chosen initial conditions are in principle consistent with a physical scenario but generate a finite net current with no fields. An EM PIC code integrates the EM fields in time by using the Ampere's and Faraday's equations only $\left(\partial_{t} \mathbf{E}=\nabla \times \mathbf{B}-4 \pi \mathbf{J}\right.$ and $\left.\partial_{t} \mathbf{B}=-\nabla \times \mathbf{E}\right)$. Running a simulation with an initial net current generates oscillations that are not very critical for the evolution of the system (e.g., the generation of all the relevant waves is preserved) but worsen the analysis and in particular the estimation of the energy balance (see also a discussion in Thurgood \& Tsiklauri, 2015).

In our simulations the background density is composed in fact of two populations of electrons: a "return" population that enables the total current to be $0\left(n_{r}=10 n_{b} v_{r}=-v_{b} / 10\right)$ and the "bulk" having zero net velocity and density $n_{\text {bulk }}=n_{0}-n_{r}$.

\section{A2. Spatial Resolution and Number of Particles Per Cell}

The problem of the resolution on this kind of simulation is connected to the numerical heating that can affect PIC codes and usually leads to consider higher resolution (i.e., $\Delta x \sim \lambda_{D}$ ) to be on the safe side. We ran several tests using smaller boxes with resolutions up to $\Delta x \sim \lambda_{D}$ without noticing discrepancies with the results obtained with $\Delta x \sim 3 \lambda_{D}$. Moreover, we checked carefully if an increase of the bulk temperature was to be observed and we did not measure any. Our results are in agreement with a recent investigation performed by (Arber et al., 2015). They show how the numerical temperature growth is inversely proportional to the number of particles per cell and depends on the interpolation function used. In our case, the resolution is sufficient for the simulation run to be numerically stable, given the very high number of particles per cell and the second-order interpolation function.

\section{A3. Fourier Analysis, Grid Size, and Available Modes in the Numerical Box}

The Fourier analysis of the simulation results enables to correctly identify the waves from their dispersion relation, estimate their direction of propagation, select specific modes, and evaluate their energy content. This allows for example to distinguish static and oscillating components of each field. Practically, we considered several temporal windows of extension $L_{t}=20 f_{P}^{-1}$ with frequent outputs (every $0.2 f_{P}^{-1}$ ) to perform, on each temporal window, a 3-D spatiotemporal $(x, y, t)$ Fourier analysis. While in space the simulation box is perfectly periodic, the nonperiodicity of the temporal domain was addressed by a Hann windowing.

Regarding the required size box and spatial resolution, the simulation box both requires (i) a spatial resolution high enough (i.e., a small enough size of the grid cell) to be numerically stable to the finite grid instability (see Appendix A2) and (ii) a numerical box size high enough to contain several wavelengths of the EM radiation. A small box with periodic boundary conditions only allows for the description a small number of wave vectors. This particularly critical for long wavelengths. As a consequence, a long-wavelength EM radiation has only a handful of possible directions of propagation in the box. In this study, this can quench 

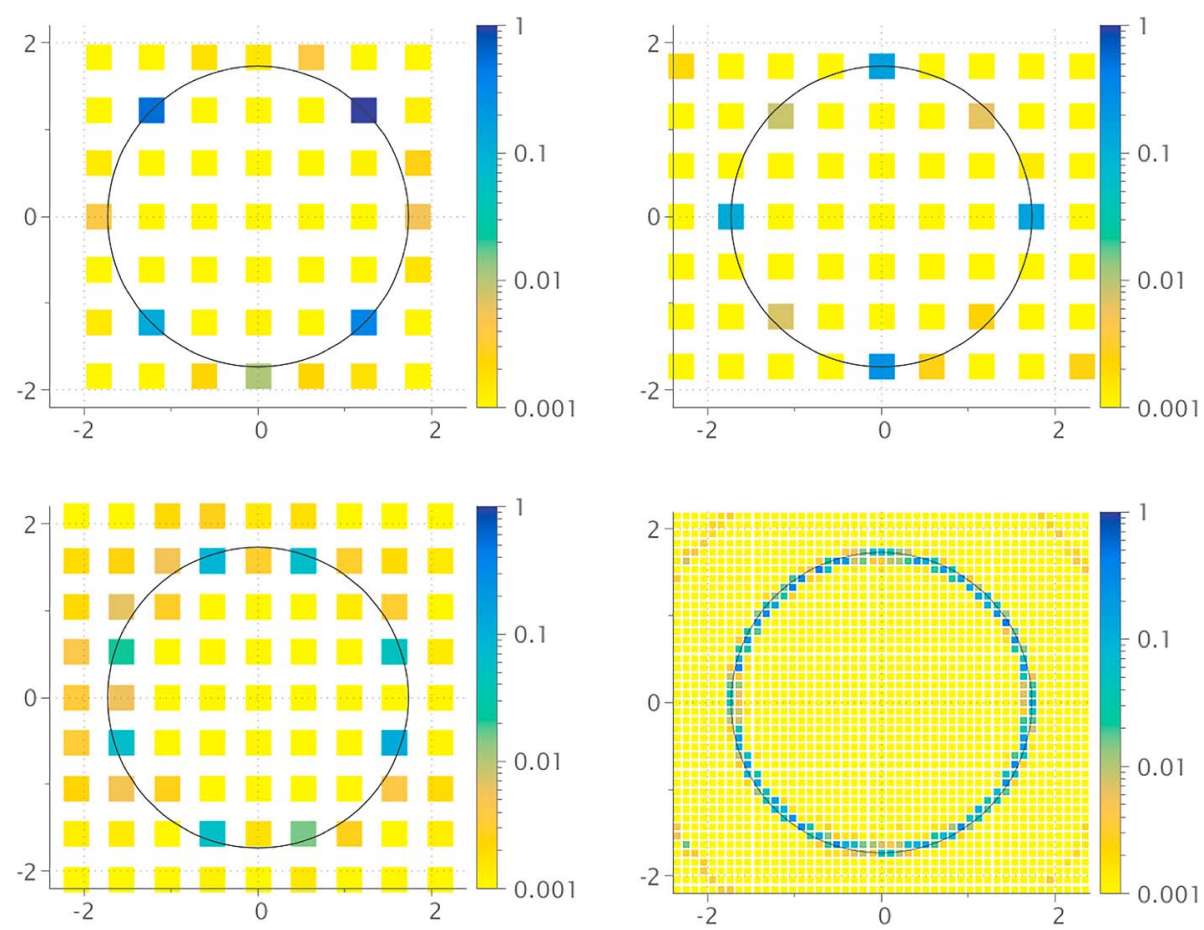

Figure A1. Spatiotemporal fast Fourier transforms of the magnetic field $\left(B_{z}\right)$ at frequency $2 \omega_{p e}$ for several simulation box size. (top left) $L x=L y=512$; (top right) $L x=L y=545$; (bottom left) $L x=L y=600$ (as in Thurgood \& Tsiklauri, 2015); (bottom right) $L x=L y=3,072$. (this work).

Acknowledgments

This work carried out within the framework of the LABEX Plas@Par benefited from a state aid managed by the National Agency of the Research (ANR) under the program "Investissements d'Avenir" bearing the reference ANR-11-IDEX-0004-02. Work at LPC2E/CNRS was supported by CNES and by ANR under the financial agreement

ANR-15-CE31-0009-01. We

acknowledge the use of computational time from projects CINECA Class B

ISCRA Projects code HP10BPGG4D.

The large amount of data produced by the different multidimensional, kinetic simulations reported in this paper could not be stored on a long-term dedicated repository because of storage constraints. However, the data used in this work can be directly duplicated through the open source,

particle-in-cell (PIC) code "piccante" (Sgattoni et al., 2015, 2013), used in this work. The source code has been made publicly available on a long-term repository at

https://github.com/ALaDyn/piccante, together with a joint User Manual and examples of input files to guide the user in reproducing the simulations. the emission mechanism and lead to an unreliable evaluation of both the energy transfer efficiency and the emission angular pattern, two key topics studied in this work. Particular care then needs to be taken when choosing the size of the box. An example is provided in Figure A1 where four different box sizes were chosen to illustrate the numerical limitations discussed above. In all cases the EM emission is resolved, however, only some directions are properly described. This gives a false impression of preferred or forbidden directions for the EM emission. For example, in the top left panel, the simulation box has been chosen so that two EM wavelengths fill the simulation box in the diagonal direction $\left(2 \lambda_{T_{2 f}}=\sqrt{L_{x}^{2}+L_{y}^{2}}\right)$, while in the top right panel, two EM wavelengths fill the simulation box in the $x$ and $y$ directions $\left(2 \lambda_{T_{2 f}}=L_{x}=L_{y}\right)$. For the production simulations, described in this paper, our choice of box size avoids any severe limitation of the direction of propagation (see bottom right panel of Figure A1). This comes to a much higher price in terms of numerical resources that are, unfortunately, absolutely necessary to provide a thorough study of the radio emission directivity and conversion efficiency.

\section{References}

Arber, T. D., Bennett, K., Brady, C. S., Ramsay, M. G., Sircombe, N. J., Gillies, P., et al. (2015). Contemporary particle-in-cell approach to laser-plasma modelling. Plasma Physics and Controlled Fusion, 57, 113001. https://doi.org/10.1088/0741-3335/57/11/113001

Bonnin, X., Hoang, S., \& Maksimovic, M. (2008). The directivity of solar type III bursts at hectometer and kilometer wavelengths: Wind-Ulysses observations. Astronomy and Astrophysics, 489, 419-427. https://doi.org/10.1051/0004-6361:200809777

Briand, C. (2015). Langmuir waves across the heliosphere. Journal of Plasma Physics, 81, 325810204. https://doi.org/10.1017/ S0022377815000112

Briand, C., Henri, P., \& Hoang, S. (2014). Inhibition of type III radio emissions due to the interaction between two electron beams: Observations and simulations. Journal of Geophysical Research: Space Physics, 119, 2365-2378. https://doi.org/10.1002/2013JA019688

Fitzenreiter, R. J., Fainberg, J., \& Bundy, R. B. (1976). Directivity of low frequency solar type III radio bursts. Solar Physics, 46, 465-473. https://doi.org/10.1007/BF00149870

Ginzburg, V. L., \& Zhelezniakov, V. V. (1958). On the possible mechanisms of sporadic solar radio emission (radiation in an isotropic plasma). Soviet Astronomy, 2, 653.

Graham, D. B., Cairns, I. H., \& Malaspina, D. M. (2014). Harmonic waves and sheath rectification in type III solar radio bursts. Journal of Geophysical Research: Space Physics, 119, 723-741. https://doi.org/10.1002/2013JA019317

Graham, D. B., Cairns, I. H., \& Robinson, P. A. (2013). Langmuir "snakes" and electrostatic decay in the solar wind. Geophysical Research Letters, 40, 1934-1939. https://doi.org/10.1002/grl.50475 
Henri, P., Briand, C., Mangeney, A., Bale, S. D., Califano, F., Goetz, K., \& Kaiser, M. (2009). Evidence for wave coupling in type III emissions. Journal of Geophysical Research, 114, A03103. https://doi.org/10.1029/2008JA013738

Henri, P., Califano, F., Briand, C., \& Mangeney, A. (2010a). Vlasov simulations of Langmuir electrostatic decay and consequences for type III observations. Twelfth International Solar Wind Conference, 1216, 288-291. https://doi.org/10.1063/1.3395857

Henri, P., Califano, F., Briand, C., \& Mangeney, A. (2010b). Vlasov-Poisson simulations of electrostatic parametric instability for localized Langmuir wave packets in the solar wind. Journal of Geophysical Research, 115, A06106. https://doi.org/10.1029/2009JA014969

Hoang, S., Poquerusse, M., \& Bougeret, J.-L. (1997). The directivity of solar kilometric type III Bursts: Ulysses-Artemis observations in and out of the ecliptic plane. Solar Physics, 172, 307-316. https://doi.org/10.1023/A:1004956913131

Kasaba, Y., Matsumoto, H., \& Omura, Y. (2001). One- and two-dimensional simulations of electron beam instability: Generation of electrostatic and electromagnetic $2 \mathrm{f}_{p}$ waves. Journal of Geophysical Research, 106, 18,693-18,712. https://doi.org/10.1029/2000JA000329

Krafft, C., Volokitin, A. S., \& Krasnoselskikh, V. V. (2015). Langmuir wave decay in inhomogeneous solar wind plasmas: Simulation results. The Astrophysical Journal, 809, 176. https://doi.org/10.1088/0004-637X/809/2/176

Kruer, W. (1988). The physics of laser-plasma interaction: New York.

Malaspina, D. M., Cairns, I. H., \& Ergun, R. E. (2010). The $2 \mathrm{f}_{p}$ radiation from localized Langmuir waves. Journal of Geophysical Research, 115, A01101. https://doi.org/10.1029/2009JA014609

Malaspina, D. M., Graham, D. B., Ergun, R. E., \& Cairns, I. H. (2013). Langmuir wave harmonics due to driven nonlinear currents. Journal of Geophysical Research: Space Physics, 118, 6880-6888. https://doi.org/10.1002/2013JA019309

Melrose, D. B. (1973). Predicted preferential backward emission at the second harmonic of the plasma frequency in solar radio bursts. The Astrophysical Journal, 15, 55.

Montgomery, M. D., Bame, S. J., \& Hundhausen, A. J. (1968). Solar wind electrons: Vela 4 measurements. Journal of Geophysical Research, 73(15), 4999-5003. https://doi.org/10.1029/JA073i015p04999

Poquérusse, M., Hoang, S., Bougeret, J.-L., \& Moncuquet, M. (1996). Ulysses-ARTEMIS radio observation of energetic flare electrons. In D. Winterhalter, J. T. Gosling, S. R. Habbal, W. S. Kurth, \& M. Neugebauer (Eds.), Proceedings of the eigth international solar wind conference: Solar wind eight, AIP Conference Proceedings (Vol. 382, pp. 62-65). American Institute of Physics. https://doi.org/10.1063/1.51360

Rhee, T., Ryu, C.-M., Woo, M., Kaang, H. H., Yi, S., \& Yoon, P. H. (2009). Multiple harmonic plasma emission. The Astrophysical Journal, 694, 618-625. https://doi.org/10.1088/0004-637X/694/1/618

Riconda, C., Weber, S., Tikhonchuk, V. T., \& Héron, A. (2011). Kinetic simulations of stimulated Raman backscattering and related processes for the shock-ignition approach to inertial confinement fusion. Physics of Plasmas, 18, 092701.

Robinson, P. A., \& Cairns, I. H. (1998). Fundamental and harmonic emission in type III solar radio bursts-II. Dominant modes and dynamic spectra. Solar Physics, 181, 395-428. https://doi.org/10.1023/A:1005033015723

Robinson, P. A., Willes, A. J., \& Cairns, I. H. (1993). Dynamics of Langmuir and ion-sound waves in type III solar radio sources. The Astrophysical Journal, 408, 720-734. https://doi.org/10.1086/172632

Schleyer, F., Cairns, I. H., \& Kim, E.-H. (2014). Linear mode conversion of Langmuir/z mode waves to radiation: Averaged energy conversion efficiencies, polarization, and applications to Earth's continuum radiation. Journal of Geophysical Research: Space Physics, 119, 3392-3410. https://doi.org/10.1002/2013JA019364

Sgattoni, A., Amiranoff, F., Briand, C., Henri, P., Grech, M., \& Riconda, C. (2017). Beam-plasma instability and density holes: Langmuir wave-packet formation and particle acceleration. Physics of Plasmas, 24(7), 072103. https://doi.org/10.1063/1.4989724

Sgattoni, A., Fedeli, L., \& Sinigardi, S. (2013). Piccante: An open-source, fully relativistic, massively parallel particle-in-cell code. Retrieved from http://aladyn.github.io/piccante/

Sgattoni, A., Fedeli, L., Sinigardi, S., Marocchino, A., Macchi, A., Weinberg, V., \& Karmakar, A. (2015). Optimising PICCANTE-An open source particle-in-cell code for advanced simulations on Tier-0 systems. ArXiv e-prints.

Takakura, T. (1979). Dynamics of a cloud of fast electrons travelling through the plasma. IV: Simulation for type III solar radio bursts. Solar Physics, 61, 161-186. https://doi.org/10.1007/BF00155454

Thurgood, J. O., \& Tsiklauri, D. (2015). Self-consistent particle-in-cell simulations of fundamental and harmonic plasma radio emission mechanisms. Astronomy and Astrophysics, 584, A83. https://doi.org/10.1051/0004-6361/201527079

Thurgood, J. O., \& Tsiklauri, D. (2016). Particle-in-cell simulations of the relaxation of electron beams in inhomogeneous solar wind plasmas. Journal of Plasma Physics, 82(6), 905820604. https://doi.org/10.1017/S0022377816000970

Timofeev, I. V. (2012). Two-dimensional simulations of nonlinear beam-plasma interaction in isotropic and magnetized plasmas. Physics of Plasmas, 19(4), 042108. https://doi.org/10.1063/1.3700441

Umeda, T. (2010). Electromagnetic plasma emission during beam-plasma interaction: Parametric decay versus induced scattering. Journal of Geophysical Research, 115, A01204. https://doi.org/10.1029/2009JA014643

Weber, S., \& Riconda, C. (2015). Temperature dependence of parametric instabilities in the context of the shock ignition approach to inertial confinement fusion. High Power Laser Science and Engineering, 3, e6.

Weber, S., Riconda, C., Klimo, O., Héron, A., \& Tikhonchuk, V. T. (2012). Fast saturation of the two-plasmon-decay instability fr shock-ignition conditions. Physical Review E, 85, 016403.

Ziebell, L. F., Yoon, P. H., Gaelzer, R., \& Pavan, J. (2014). Plasma emission by weak turbulence processes. The Astrophysical Journal, 795, 320. https://doi.org/10.1088/2041-8205/795/2/L32

Ziebell, L. F., Yoon, P. H., Petruzzellis, L. T., Gaelzer, R., \& Pavan, J. (2015). Plasma emission by nonlinear electromagnetic processes. The Astrophysical Journal, 806(2), 237. 